\title{
SENSITIVITY TO BENZYLPENICILLIN OF N.MENINGITIDIS ISOLATED FROM CHILDREN UNDER 5 YEARS WITH INVASIVE BACTERIAL
}

DISEASES

H. Kharkhal, L. Titov.

The Republican Research and Practical Center for Epidemiology and Microbiology, Laboratory of Clinical and Experimental Microbiology, Minsk, Belarus.

\section{Background}

Meningococcal infection is an invasive bacterial disease caused by N.meningitidis. in particular, are still actual not only among children infections. Despite the fact that Neisseria spp. are generally sensitive to penicillins, nowadays we have a rise of antimicrobial resistance. That's why it is important to study antimicrobial sensitivity rates of N.meningitidis, especially to drugs of choice, like benzylpenicillin, ampicillin carbapenems, chloramphenicol.

\section{Methods}

28 N.meningitidis isolates were collected from blood $(n=9)$ and CSF $(n=19)$ of children with invasive bacterial diseases under 5 years from Brest region $(n=10)$, Minsk ( $n=9)$, Grodno and Mogilev regions $(n=3$ each), Vitebsk region $(n=2)$, Minsk region $(n=1)$ for the period of 2011-2017.

Isolates were tested on Muller-Hinton agar with $20 \%$ horse blood serum by E-tests strips with benzylpenicillin concentration 0.002-32 $\mu \mathrm{g} / \mathrm{mL}$. Results interpreted according to European Committee on Antimicrobial Susceptibility Testing (EUCAST) breakpoint tables for interpretation of MICs and zone diameters, version 7.0, valid from 2017-01-01, where sensitive category $\mathrm{MIC} \leq 0,06$, intermediate category $\mathrm{MIC}>0,06$ and $\leq 0,25$, resistant category MIC>0,25.

N.meningitidis serogroup was determined by specific genes detection (synD serogroup $B$, synE - serogroup $C, \operatorname{sac} B$ serogroup $A, x c b B$ - serogroup $X, \operatorname{syn} G$ serogroup W135) by the real-time PCR. Allel of house-keeping gene pdhC was determined by MLST reaction.

\section{Results}

1. N.meningitidis isolates characterization

28 isolates of N.meningitidis were serogroups $B$ $(n=24), \quad C \quad(n=2), W 135 \quad(n=1)$ and 1 non-type isolate.

The dominant serogroup is $B$, which was detected in 16 CSF samples (from Brest region (6 cases), Minsk (4), Grodno region (3), Mogilev region (2) and 1 from Vitebsk region) and 8 blood samples (Brest region (4), Minsk (3) and 1 from Mogilev region).

Serogroup C occurred twice in CSF and blood, both from Minsk. Serogroup W135 was detected in CSF in Vitebsk region. 1 isolate from Minsk region was non-type. 1 N.meningitidis type B had a new allel of house-keeping gene pdhC, firstly assigned as 888 (obtained from CSF of 1 years old girl, Brest region).

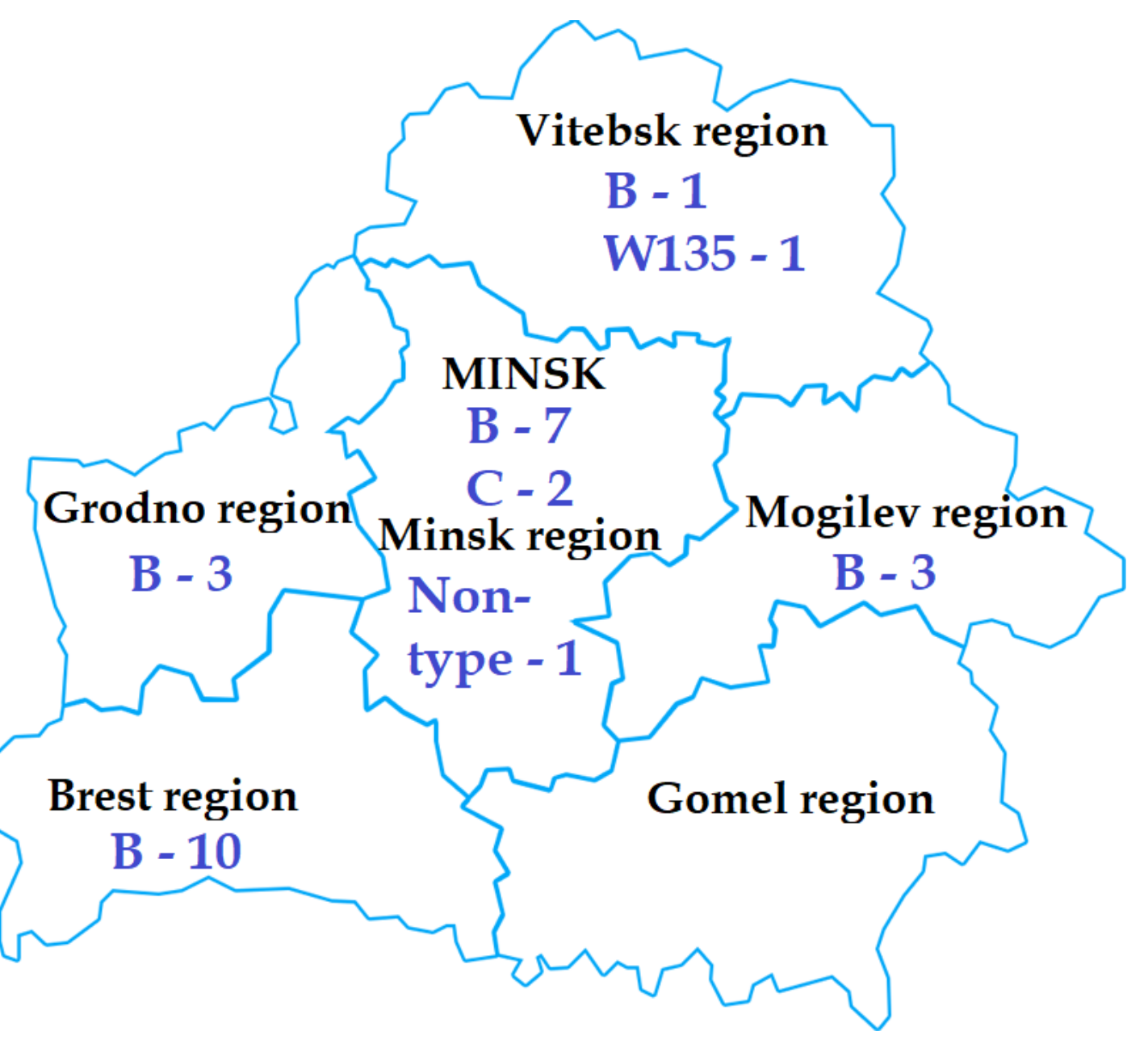

Figure 1 Number of N.meningitidis isolates and serogroups distribution in regions Republic of Belarus

\section{N.meningitidis sensitivity to benzylpenicillin}

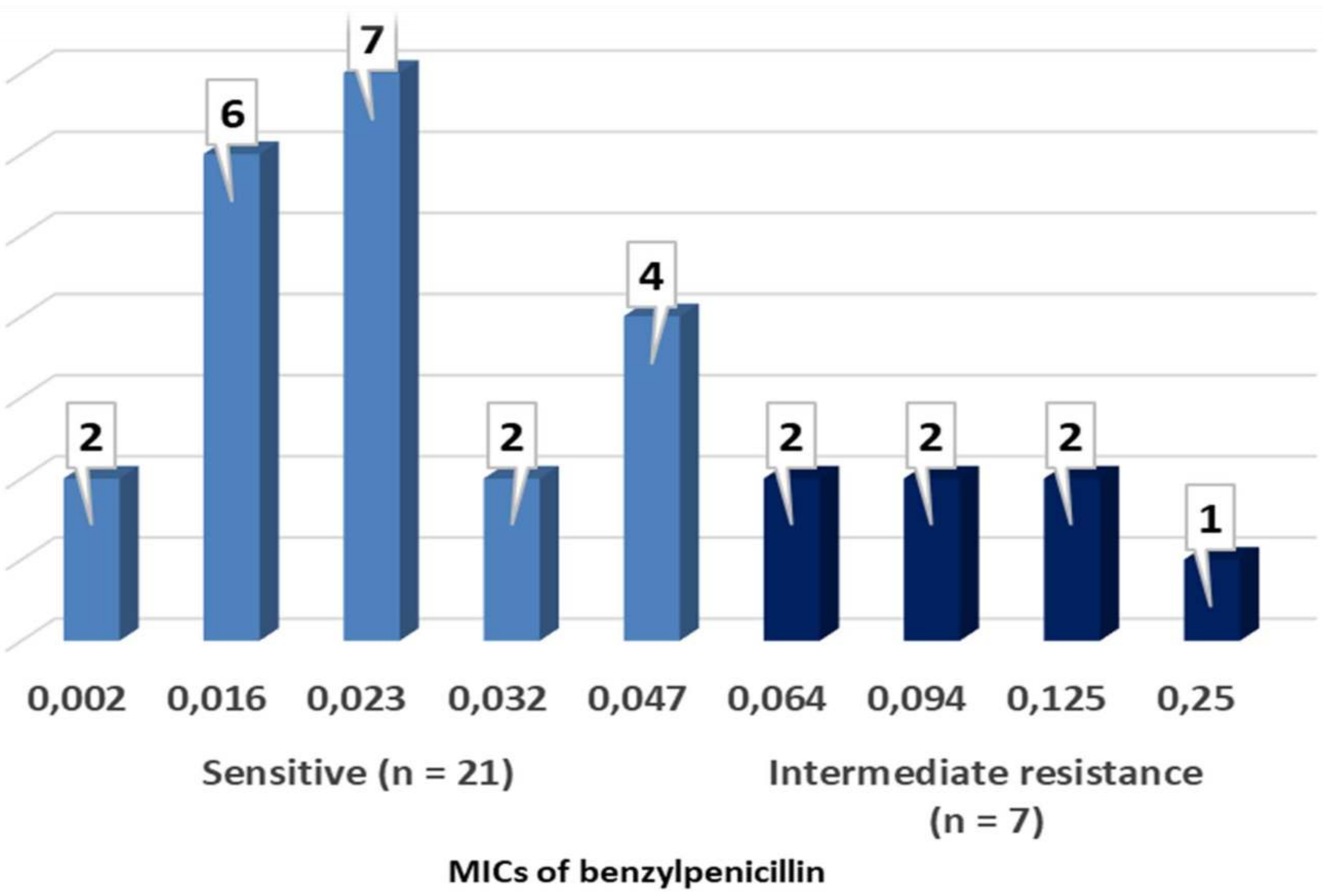

Figure 2 Sensitivity of 28 N.meningitidis isolates to benzylpenicillin

21 isolates of N.meningitidis were sensitive to benzylpenicillin. MICs of sensitive isolates: $0.002(n=2), 0.016(n=6), 0.023$ $(n=7), 0.032(n=2)$ and $0.047(n=4)$. The serotype of sensitive N.meningitidis was $B$ ( $n=18$, all MICs), $C(n=1, M I C=0.032), W 135$ $(\mathrm{n}=1, \mathrm{MIC}=0.047)$ and one none-type isolate with MIC 0.002. 7 isolates had intermediate sensitivity with next MICs: $0.064(\mathrm{n}=2)$, $0.094(n=2), 0.125(n=2), 0.25(n=1$, which is also had intermediate sensitivity to ampicillin and amoxicillin). One isolate was $N$.meningitidis $\mathrm{C}$ with $\mathrm{MIC}=0.094$. Other N.meningitidis $\mathrm{B}$.

\section{Conclusions}

The dominant serogroup of N.meningitidis isolated from children under 5 years with invasive bacterial diseases is B in general, and in an intermediate group of sensitivity to benzylpenicillin in particular.

antibiotics of the penicillin class are still widely used in clinical practice for the treatment of meningococcal infections. Thus, despite of the fact that there are no strongly resistant isolates to benzylpenicillin,, isolates with intermediate sensitivity exists quite often (25\%). 